\title{
МОДЕЛЬ ПЕРЕХОДНЫХ ПРОЦЕССОВ В СКВАЖИНЕ С ЧАСТОТНО-РЕГУЛИРУЕМЫМ ЭЛЕКТРОЦЕНТРОБЕЖНЫМ НАСОСОМ
}

\section{Соловьев Илья Георгиевич',} solovyev@ikz.ru

\author{
Говорков Денис Александрович ${ }^{1,2}$, \\ dagovorkov@mail.ru \\ Белашевский Сергей Сергеевич, \\ s.belashevsky@gmail.com

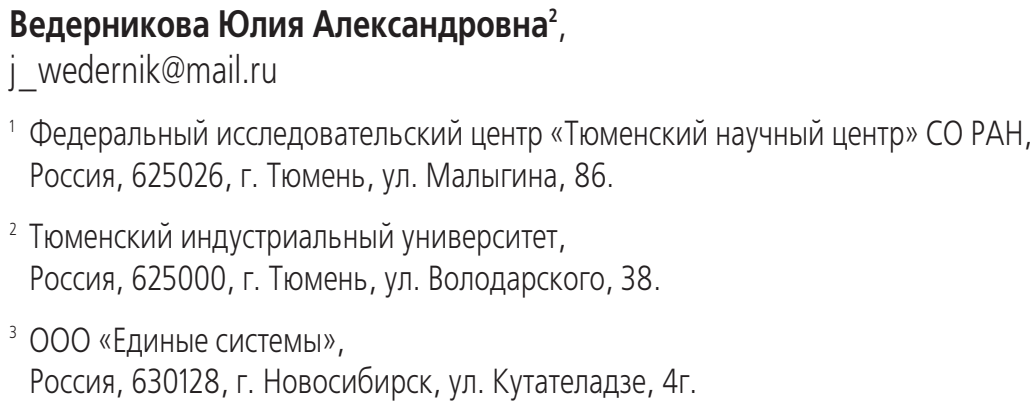

Актуальность исследования связана с проблемой эффективности контроля динамических режимов эксплуатации скважин, оснащённых частотно-регулируемым электроцентробежным насосом, при создании и вычислительной реализации моделей скважинной системы, работающих в режиме реального времени. Комплексная функциональность представленного в статье решения позволяет воспроизводить реальные формы поведения системы в переходных и равновесных режимах работы в условиях действий осложняющих факторов, что и является основой корректного параметрического оценивания с использованием данных натурного контроля режимных состояний.

Цель: построение комплексной гидродинамической модели типа «пласт-скважина-электронасос-устье», ориентированной на задачи диспетчерского контроля осложнённых режимов эксплуатации систем в условиях реального времени.

Методы: материального баланса, гидроупругой линейной фильтрации, гидростатики, линеаризованной кинетики потерь на трение, численного моделирования дифференциальных уравнений.

Результаты. Сконструированная и численно реализованная гидродинамическая модель скважины с электроцентробежным насосом воспроизводит целостную, логически обусловленную картину реального поведения системы в переходных и равновесных режимах эксплуатации в условиях действия осложняющих факторов. Упрощенный характер описания образующих компонент и связей позволяет реализовывать и применять функционально обновлённые инструменты контроля по месту в рамках информационных ресурсов диспетчерских служб предприятия.

Выводы. Комплексность и факторная полнота описания моделей являются основой конструирования алгоритмов и регламентов параметрической идентификации и последующего опознавания видов и уровней осложнений по данным реальной эксплуатации. Реализация вычислительного процесса в темпе с динамикой обновления данных контроля состояний и возмущений позволит использовать модель как виртуальный измеритель расширенного вектора состояния скважины в переходных и установившихся режимах работы.

\section{Ключевые слова:}

Гидродинамика притока, подъёмник, электроцентробежный насос, модель, гидростатические потери напора, продуктивность, осложняющие факторы.

\section{Введение}

Современные стандарты $[1,2]$ обустройства скважин средствами глубинного контроля и регулирования расширяют потенциалы эффективности новых регламентов управления процессами эксплуатации нефтедобывающих систем. Ожидаемый эффект во многом связан $[3,4]$ с созданием и цифровой реализацией автоматизированной технологии контроля гидродинамических параметров и состояний скважинной системы (CС) в условиях реального времени [5]. Разработка подобных решений основана на построении гидродинамической модели скважины [6], отражающей взаимовлияния составных компонентов системы и действия внешних плохо предсказуемых факторов эксплуатации $[7,8]$. Основные компоненты скважины, обустроенной электроцентробежный насос (ЭЦН), включают:

- гидродинамику притока;

- кинетику и гидростатику потерь напора в сегментах подъёмника: низ обсадной колоны (OK), затрубное пространство (ЗТ), насосно-компрессорная труба (НКТ);

- напорную характеристику частотно-регулируемого насоса;

- параметры и состояния устья.

Внешние по отношению к СС возмущающие факторы можно классифицировать как 
- возмущения параметров притока: вариации среднепластового давления - $p_{R}$ на контуре питания скважины, дрейф коэффициента продуктивности $-w_{R}$, рост обводнённости - $\beta$ добываемой продукции;

- рост гидросопротивлений сегментов конструкции вследствие сорбирования парафинов, солей, механических примесей и прочих полютантов;

- снижение напора насоса вследствие абразивного износа;

- негативные проявления свободного газа из-за разгазирования нефти в насосе и на забое скважины.

Большее многообразие видов возмущений связывается с особенностями строения и работы притока скважины, а именно: одновременное дренирование нескольких горизонтов, неравномерность и неоднородность по фазному составу притока к горизонтальным сегментам забоя скважины, влияние конусов подошвенной воды и газа, прочие особенности конструкций и режимов работы. Детализация подобного класса возмущений в большей степени отвечает проблемам управления разработкой залежи и не входит в круг обсуждаемых ниже тем. С другой стороны, выделенный компонентный состав модели системы и виды осложнений соответствуют функционалу задач контроля процессов эксплуатации СС со стороны диспетчерских служб предприятия. Именно для данного класса задач контроля конструируется гидродинамическая модель скважины с ЭЦН. Фондоёмкие инструменты интегрированного термобарического анализа процессов и состояний нефтепромысловых систем $[9,10]$, реализуемые в виде информационно аналитических центров компаний $[11,12]$, плохо подходят для задач оперативного контроля режимов в изменяющихся условиях эксплуатации. Система диспетчерского контроля и соответствующие инструменты модельного анализа и ситуационного прогнозирования целесообразно реализовывать по месту сбора первичной информации в рамках информационных ресурсов предприятия. Упрощения, связанные с переходом от пространственной детализации классического распределённого анализа к кусочно-линейным корреляциям между состояниями системы в ключевых точках подъёмника, с учётом балансовых равенств и факторов внешних возмущений, позволяет корректно воспроизводить графики состояния системы в равновесных и переходных режимах и надёжно выделять первопричины отклонений состояний от расчетных значений.

\section{Барометрическая модель скважинной системы}

На рис. 1 изображена функциональная схема скважины с ЭЦН, приведённая к вертикали, и соответствующий график распределения давлений по стволу скважины с включённым: $u=1$, и выключенным: $u=0$ насосом. Обозначения параметров и переменных состояния системы на схеме поясня- ются в тексте по ходу изложения, единицы измерений указаны в таблице моделируемых примеров (таблица).

Модель притока представлена зоной окаймления пласта с давлением подпора - $p_{R}$, призабойной зоной (ПЗ) со средним давлением $-p(2, t)$ и забоем скважины с давлением - $p(1, t)$. Данная модель устанавливает связь между объёмными притоками жидкости в ПЗ $-q(2, t)$ и забой скважины $-q(t)$ в зависимости от перепада давлений в объявленных зонах. Для простоты обозначений явная зависимость переменных состояния от времени $-t$ там, где это идёт без ущерба для понимания, будет опускаться. Основываясь на линейном законе упругой фильтрации [13-15], выписанном для выделенных конечных зон пласта, имеем

$$
\begin{gathered}
\tau(2) \frac{d p(2)}{d t}=q(2)-q, \\
q(2)=w(2)\left(p_{R}-p(2)\right), \\
q=\left(1-\beta_{G}(1)\right) w(1)(p(2)-p(1)),
\end{gathered}
$$

где $\tau(2)$ - параметр объёмной гидроупругости ПЗ, а $w(1), w(2)$ - гидропроводности переходов «пласт-ПЗ» и «ПЗ-забой» по жидкости. В условиях разгазирования нефти на забое скважины, когда давление $p(1)$ становится ниже давления насыщения $p_{G 0}$, продуктивность притока по жидкости уменьшается $(1-\beta(1)) w(1)$, за счёт частичного перекрытия фильтруемой зоны забоя свободным газом - $q_{G}(1)$ (не отождествлять с прорывом конуса газа из газовой шапки). По аналогии с [16], объёмная доля газа на забое - $\beta_{G}(1)$, определяется выражением

$$
\begin{gathered}
\beta_{G}(1)=\frac{q_{G}(1)}{q_{G}(1)+q}=\frac{\mu_{R}\left(p_{G O}-p(1)\right)_{+}}{p(1)+\mu_{R}\left(p_{G O}-p(1)\right)_{+}}, \\
\mu_{R}=(1-\beta) G \frac{p_{0}}{p_{G O}-p_{0}},
\end{gathered}
$$

что следует из линейной аппроксимации долевой функции $\varphi_{G}(1)=\frac{\left(p_{G O}-p(1)\right)_{+}}{p_{G O}-p_{0}}$ массы газа $m_{G}(1)=$ $=Y_{G} \varphi_{G}(1) q(1)$, вышедшего при давлении $p(1)$ в свободное состояние, с весовой оценкой [17] потока $Y_{G}=(1-\beta) \gamma_{G 0} G$. Здесь $G-$ газовый фактор нефти; $\gamma_{G 0}=\chi_{G} p_{0}-$ удельный вес газа в нормальных термобарических условиях; $\chi_{G}-$ настроечный, с учетом $p V T$-диаграммы, параметр; $p_{0}=0,1 \mathrm{MПа}$ - давление атмосферное.

Модель «низа» колоны определяет потерю напора в интервале от забоя скважины на уровне перфорации $-H_{R}$, с давлением - $p(1)$, до уровня подвески насоса $-H_{N}$, с давлением на приеме $-p(3)$.

$$
p(1)=p(3)+\gamma\left(H_{R}-H_{N}+r_{K} q\right),
$$

где $\gamma=(1-\beta) \gamma_{0}+\beta \gamma_{W}-$ удельный вес газо-жидкостной смеси, средний по стволу скважины, с долевыми компонентами: $\gamma_{0}$ - удельный вес газированной нефти и $\gamma_{W}-$ удельный вес попутной воды; $r_{K}=\rho_{K}\left(H_{R}-H_{N}\right)$ - гидросопротивление линеаризо- 


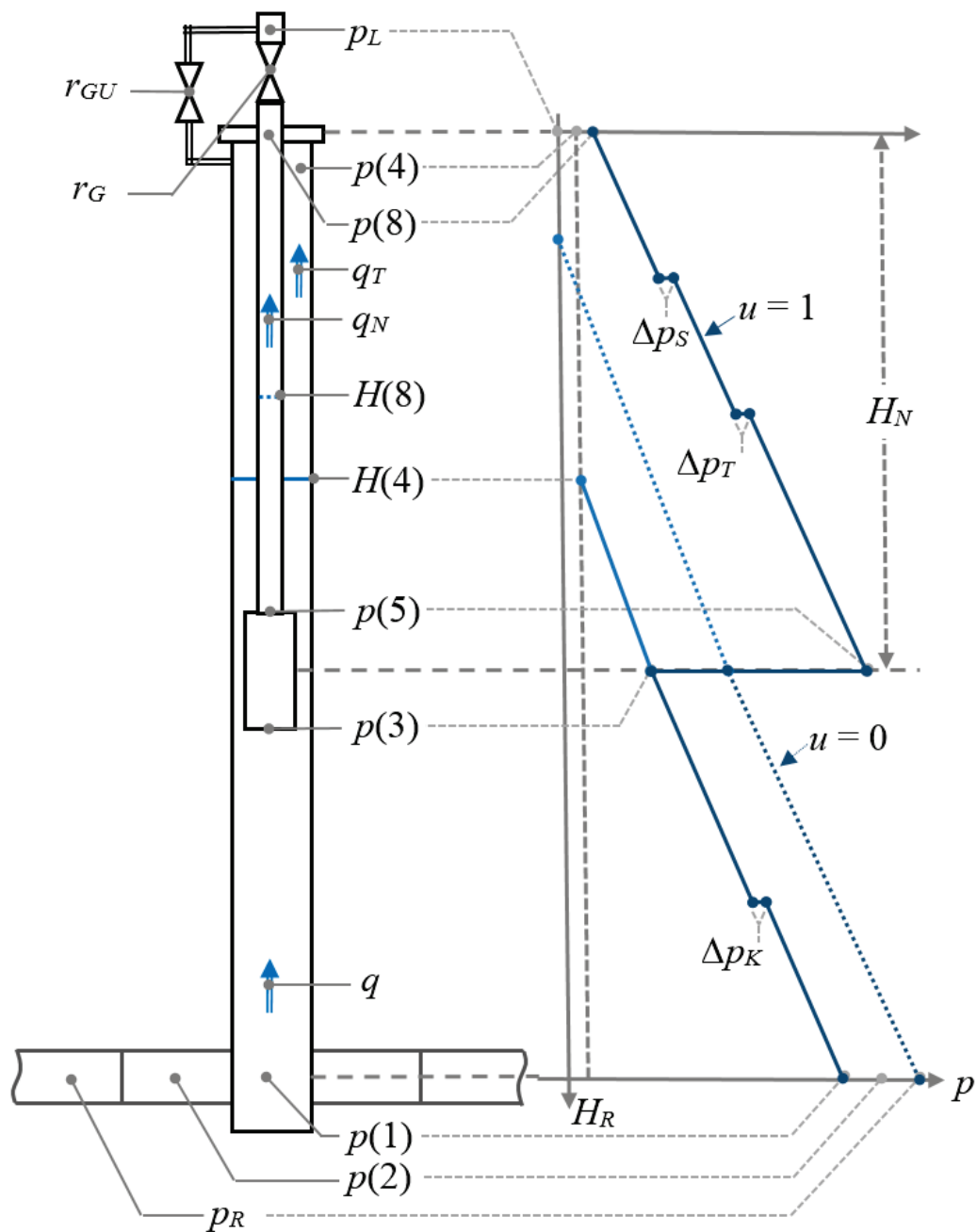

Pис. 1. Функциональная схема скважины с ЭЦН (Аp- потери напора на трение в соответствующих участках подъелника)

Fig. 1. Functional diagram of the ESP-equipped well ( $\Delta p$ is the pressure loss on friction in the corresponding sections of the lift)

ванной модели кинетических потерь на трение ( $\rho_{K}-$ настроечный коэффициент удельных потерь). Основная гидростатическая доля потерь приходится на вес столба газожидкостной смеси, а малые кинетические потери напора, аппроксимируемые линейной формой, зависят от объёмного расхода прокачиваемой жидкости - $q$.

По аналогичной схеме строится модель потерь напора в НКТ в интервале от выкида насоса, с давлением - $p(5)$, до устьевого штуцера, с давлениeм $-p(8)$ :

$$
p(5)=p(8)+\gamma\left(H_{N}-H(8)+r_{N S} q_{N}\right) .
$$

Здесь $q_{N}-$ объёмный расход жидкости в НКТ, $r_{N S}=r_{N}+r_{S}$ - суммарное гидросопротивление кинетических потерь в сегменте подъёмника, составленное из $r_{N}=\rho_{N} H_{N}$ сопротивления НКТ и дополненное сопротивлением потерь $r_{S}$ от сорбирования солей и прочих полютантов. В случае переходных режимов, например, при выключении ЭЦН, в НКТ складываются условия обратного стравливания жидкости $q_{N}<0$, если обратный клапан на выкиде насоса негерметичен. Учитывая подобные ситуации, в модели сегмента (4) введена переменная динамического уровня жидкости в НКТ $H(8, t)$, подчиняющаяся уравнению

$$
S_{N} \frac{d}{d t} H(8, t)=-1_{8}(t) q_{N}(t),
$$

где $S_{N}$ - площадь сечения НКТ, а $1_{8}(t)$ - идентификатор отсутствия подачи в линейный нефтесборник, определяется условием

$$
1_{8}(t)=\left\{\begin{array}{l}
1, \text { если }(H(8) \geq 0) \vee\left((H(8)=0) \wedge\left(q_{N} \leq 0\right)\right) ; \\
\left.0, \text { если }(H(8)=0) \wedge\left(q_{N}>0\right)\right) .
\end{array}\right.
$$

Погружные центробежные насосы типового исполнения критичны к избыточному объёму свободного газа на приёме первой ступени. Если объёмная доля газа у первой ступени $\beta_{G N}(t)$ превышает контрольное значение

$$
\beta_{G N}>\beta_{G}^{L I M} \approx 0,25,
$$

то велика вероятность выхода насоса в предаварийное состояние срыва подачи [18]. Для сохранения 
функциональности ЭЦН в условиях действия газового фактора, погружные установки дополнительно обустраиваются газосепараторами [19], обеспечивающими возврат до семидесяти процентов захваченного на приеме газа в затрубное пространство. В названных условиях лодель ЗТ, связывающая давления на приёме - $p(3)$ и устье $-p(4)$ (до перепускного клапана) с потоком жидкости в затрубъе, может быть представлена следующим образом

$$
p(3)=p(4)+\gamma\left(H_{N}-H(4)+\left(1-1_{4}\right) r_{T} q_{T}\right),
$$

где кинетика потерь напора по жидкости с гидросопротивлением сегмента $-r_{T}=\rho_{T} H_{N}$ начинает учитываться после заполнения и последующей циркуляции жидкости $q_{T}=q-q_{N}>0$ по затрубу, $1_{4}(t)-$ идентификатор отсутствия затрубной циркуляции, определяемый условием

$$
1_{4}(t)=\left\{\begin{array}{l}
1, \text { если }(H(4) \geq 0) \vee\left((H(4)=0) \wedge\left(q_{T} \leq 0\right)\right) ; \\
\left.0, \text { если }(H(4)=0) \wedge\left(q_{T}>0\right)\right) .
\end{array}\right.
$$

В приведённых соотношениях, как правило, ненулевое значение динамического уровня жидкости в 3 Т $H(4, t)$ (рис. 1 ) может исчезать: $H(4, t) \rightarrow 0$ с выключением ЭЦН, если изначально скважина фонтанирует. Вариации динамики уровня подчиняются уравнению

$$
S_{T} \frac{d}{d t} H(4, t)=-1_{4}(t) q_{T}(t)
$$

где $S_{T}$ - площадь сечения 3T.

Модель устьевого штуцера определяет кинетику потерь напора, если имеет место истечение жидкости через него в нефтесборник с противодавлением $p_{L}$

$$
p(8)=p_{L}+\gamma\left(1-1_{8}\right) r_{U} q_{N} .
$$

Здесь $1_{8}(t)$ - ранее определённый идентификатор отсутствия подачи в линейный нефтесборник.

В отличие от устьевого штуцера с гидросопротивлением $r_{U}$, устьевой перепускной клапан (рис. 1) с гидросопротивлением $r_{G U}$ создаёт дополнительный подпор давления в затрубъе, связанный с непрерывной подачей отсепарированной доли газа работающим насосом. Осложнение режима эксплуатации, вызванное падением уровня $H(4, t)$ ниже критической отметки срыва подачи

$$
H(4, t) \rightarrow H_{N}-h^{L I M},
$$

оказывается возможным вследствие постепенного роста давления $p$ (4) из-за перекрытия проходного сечения клапана газогидратами. Отсюда модель ус тьевого перепускного клапана может быть представлена следующим образом

$$
\begin{gathered}
p(4)=p_{L}+\gamma\left(r_{G U}+r_{G S}\right) \times \\
\times\left[\left(1-1_{4}\right) q_{T}+u k_{S} \varphi_{G}(3) \frac{Y_{G}}{\gamma}\left(q_{N}+q_{T}\right)\right],
\end{gathered}
$$

где $r_{G S}$ - дополнительное гидросопротивление, связанное с возможным осадконакоплением, $k_{S} \approx 0,7-$ коэффициент сепарации.
Модель насоса, представленная на рис. 2 трехзвенным кусочно-линейным графиком аппроксимации нормированной напорной характеристики, устанавливает связь между напором - $h_{N}$ (в метрах водяного столба), создаваемым насосом, и объёмным расходом прокачиваемой жидкости $-q_{N}$. Аналитическая форма представления модели имеет вид:

$$
\left\{\begin{array}{l}
h_{N}=u \omega^{2} v_{h} h^{0} \lambda_{0}(i)-\left(u \omega \frac{v_{h} h^{0}}{v_{q} q^{0}} \lambda_{1}(i)+1_{q} r_{P}\right) q_{N} ; \\
i=\arg \left\{q_{N} \in \omega v_{q} q^{0}[q N(i+1), q N(i)]\right\} .
\end{array}\right.
$$

Здесь пара $\left\langle q^{0}, h^{0}\right\rangle$ - соответствует номинальному режиму работы выбранного типоразмера насоса по расходу и напору; $i$ - номер участка ломаной нормированной напорной характеристики с типовыми (рис. 2), в общем случае настраиваемыми параметрами - $\left\langle\lambda_{0}(i), \lambda(i)\right\rangle ; \omega$ - относительная частота вращения вала двигателя (питающего напряжения), рассчитываемая по выражению $\omega=\Omega / \Omega_{0}$, где $\Omega$ - регулируемая угловая частота вращения вала двигателя, а $\Omega_{0}$ - номинальная угловая частота; $u=\{0,1\}=\{$ ВЫКЛ,ВКЛ $\}-$ идентификатор работы ЭЦН; $1_{q}(t)$ - идентификатор возможного противотока, определяемый условием

$$
1_{q}(t)=\left\{\begin{array}{l}
1, \text { если } q_{N} \leq 0 ; \\
0, \text { иначе. }
\end{array}\right.
$$

$r_{P}$ - гидросопротивление негерметичного обратного клапана на выкиде насоса; $\left\langle v_{h}, v_{q}\right\rangle$ - параметры деформации рабочей характеристики насоса по шкалам напора и расхода вследствие износа и засорения.

Замыкая приведённые соотношения (1)-(12) балансовым равенством «нагрузка=напор»

$$
\frac{1}{\gamma}(p(5)-p(3))=h_{N},
$$

приходим в итоге к искомой динамической модели СС с частотно-регулируемым ЭЦН. Изображённая на рис. 3 блок-схема модели фиксирует функционально-разделённую структуру данных исходных параметрических настроек, необходимых для запуска вычислительного процесса, входные управляющие воздействия, функционально разнесённые векторы возмущений и выходных состояний, воспроизводимых в процессе моделирования.

\section{Вычислительный анализ}

Перейдём к результатам вычислительного анализа динамики переходных режимов работы скважины с параметрами из таблицы. В первом варианте рассматривается скважина, обеспечивающая фонтанный режим добычи с $q_{N}=14 \mathrm{~m}^{3} /$ сут, $q_{T}=17 \mathrm{~m}^{3} /$ сут при выключенном насосе и подачу $q_{N}=81 \mathrm{~m}^{3} /$ сут при включённом ЭЦН с номинальной относительной частотой вращения $\omega 1$. Во втором скважина без фонтанирования. Рассматриваются режимы пуска после длительного останова и последующей вариации относительной частоты. 
Графики переходных процессов для указанных моделей (рис. 4,5$)$ представляют собой результаты моделирования состояний скважины при последовательных пуске, останове, повторном пуске и вариации частоты вращения работающего насоса. Временные интервалы моделирования на каждом из указанных режимов выбраны так, что система выходит на стационарный режим по переменным состояния ближе к концу интервала. На представленных далее графиках выделены наиболее интересные с точки зрения анализа временные диапазоны и моменты времени. В первом случае, на рис. 4 , воспроизводятся переходные процессы при пусках и остановах ЭЦН фонтанирующей скважины, где отмечены следующие моменты времени и состояния.

1. Останов скважины из стационарного рабочего состояния. Начиная с момента $t_{1}=10,005$ (сут) динамический уровень $H(4, t)$ в ЗТ начинает подниматься (затруб заполняется жидкостью с притока), а в НКТ вследствие стравливания жидкости через негерметичный обратный клапан ЭЦН образуются «пустоты» $H(8, t)>0$.

2. При $t_{2}=10,218$ забойное давление становится выше давления насыщения и влияние свободного газа на приток исчезает.

3. При $t_{3}=10,221$ уровни ГЖС в ЗТ и НКТ выравниваются, и далее происходит их совместное движение в сторону устья.

4. Вследствие разного гидросопротивления сегментов подъёмника полное заполнение 3Т происходит раньше $t_{41}=10,324$, чем НКТ $t_{42}=10,334$. Включение дополнительного сопротивления перепускного клапана $r_{G U}$ в канал циркуляции $q_{T}\left(t_{41}\right)>0$ снижает интенсивность подачи из $\mathrm{BT}$, активизируя заполнение НКТ. После заполнения НКТ и подключения устьевого штуцера с гидросопротивлением $r_{U}$ происходит вторичное перераспределение расходов в соответствии с суммарными сопротивлениями каналов подъемника при одинаковом подпоре со стороны забоя.

5. В отличие от момента останова $t_{1}=10,005$, когда объёмы притока и стравливания из НКТ поступают в 3Т, обеспечивая положительный скачок $q_{T}\left(t_{1}\right)>0$, вторичный запуск скважины $t_{5}=20$ со стационарного состояния останова интенсифицирует обратный забор жидкости из $3 \mathrm{~T} q_{T}\left(t_{5}\right)>0$, обеспечивая момент повышенной подачи насоса $q_{T}\left(t_{5}\right)>0$ при пуске.

таблица. Параметры моделируемой скважинной систелы Table. $\quad$ Parameters of the simulated well system

\begin{tabular}{|c|c|c|}
\hline $\begin{array}{l}\text { Наименование, ед. изм. } \\
\text { Name, units }\end{array}$ & $\begin{array}{c}\text { Величина } \\
\text { Value }\end{array}$ & $\begin{array}{l}\text { Обозначение } \\
\text { Notation }\end{array}$ \\
\hline \multicolumn{3}{|c|}{ Фонтанирующая скважина/Fountain well } \\
\hline $\begin{array}{l}\text { Среднепластовое давление, МПа } \\
\text { Average reservoir pressure, MPa }\end{array}$ & 28 & $p_{R}$ \\
\hline Глубина забоя по вертикали, м/Well depth, m & 3000 & $H_{R}$ \\
\hline $\begin{array}{l}\text { Коэффициент гидропроводности притока } \\
\text { из пласта в ПЗ скважины, } \mathrm{M}^{3} / \mathrm{M \Pi а} \text { суут } \\
\text { Reservoir productivity coefficient, } \mathrm{m}^{3} / \mathrm{MPa} \text { day }\end{array}$ & 12 & $w(1)$ \\
\hline $\begin{array}{l}\text { Коэффициент гидропроводности притока } \\
\text { из ПЗ в забой скважины, м³ } / \text { МПа·сут } \\
\text { Inflow productivity coefficient, } \mathrm{m}^{3} / \mathrm{MPa} \text { day }\end{array}$ & 60 & $w(2)$ \\
\hline Объёмная доля воды, ед./Water cut, units & 0,3 & $\beta$ \\
\hline \multicolumn{3}{|c|}{\begin{tabular}{|l|l} 
Удельный вес, MПа/м /Specific gravity, MPa/m & \\
\end{tabular}} \\
\hline · нефти/oil & $0,68 \cdot 10^{-2}$ & $\gamma_{0}$ \\
\hline · воды/water & $1,1 \cdot 10^{-2}$ & $\gamma_{W}$ \\
\hline · газа/gas & $0,8 \cdot 10^{-5}$ & $\gamma_{G O}$ \\
\hline Газовый фактор, ед./Gas factor, units & 100 & $G$ \\
\hline $\begin{array}{l}\text { Давление насыщения, МПа } \\
\text { Saturation pressure, MPa }\end{array}$ & 25 & $p_{G O}$ \\
\hline $\begin{array}{l}\text { Давление в линии нефтесбора, МПа } \\
\text { Oil collector line pressure, MPa }\end{array}$ & 1,5 & $p_{L}$ \\
\hline \multicolumn{3}{|l|}{$\begin{array}{l}\text { Гидросопротивления, }{ }^{3} /(\text { МПа·сут) } \\
\text { Hydroresistances, }{ }^{3} /(\mathrm{MPa} \cdot \text { day })\end{array}$} \\
\hline · обсадной колоны/casing & 0,2 & $r_{K}$ \\
\hline$\cdot$ HKT/tubing & 1,6 & $r_{N}$ \\
\hline · затрубного пространства/annulus & 0,8 & $r_{T}$ \\
\hline · устьевого штуцера/wellhead tubing choke & 2 & $r_{U}$ \\
\hline $\begin{array}{l}\text { перепускного клапана } \\
\text { wellhead annulus choke }\end{array}$ & 3 & $r_{G U}$ \\
\hline \multicolumn{3}{|l|}{ Параметры насоса/Pump parameters } \\
\hline · уровень подвески, м/pump depth, m & 2000 & $H_{N}$ \\
\hline · номинальный напор, м/nominal head, m & 1700 & $h^{0}$ \\
\hline \begin{tabular}{l|} 
номинальный дебит, ${ }^{3} /$ сут \\
nominal rate, $\mathrm{m}^{3} /$ day
\end{tabular} & 60 & $q^{0}$ \\
\hline \multicolumn{3}{|c|}{ Скважина без фонтанирования/Well without fountain } \\
\hline $\begin{array}{l}\text { Среднепластовое давление, } \mathrm{MПа} \\
\text { Average reservoir pressure, } \mathrm{MPa}\end{array}$ & 21 & $p_{R}$ \\
\hline $\begin{array}{l}\text { Давление насыщения, МПа } \\
\text { Saturation pressure, } \mathrm{MPa}\end{array}$ & 18 & $p_{G O}$ \\
\hline
\end{tabular}

6. В момент времени $t_{6}=20,11$ забойное давление $p\left(1, t_{6}\right)$ становится ниже давления насыщения $p_{G 0}$ и приток вновь входит в нелинейные условия действия газового фактора.

\begin{tabular}{|c|c|c|c|}
\hline$i$ & 1 & 2 & 3 \\
\hline$q N$ & $5 / 3$ & $4 / 3$ & $2 / 3$ \\
\hline$\lambda_{0}$ & $10 / 3$ & 2 & $3 / 2$ \\
\hline$\lambda_{1}$ & 2 & 1 & $1 / 4$ \\
\hline
\end{tabular}

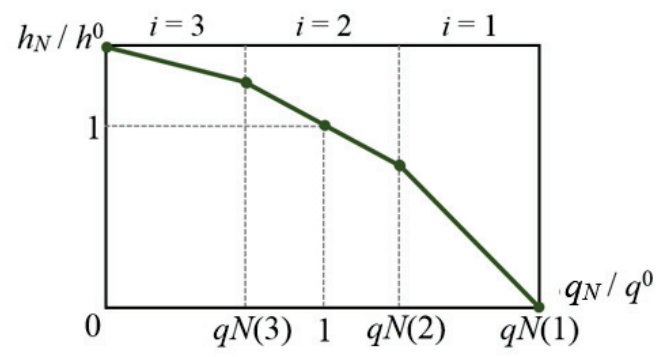

Puс. 2. График и таблица параметров нормированной напорной характеристики насоса $q_{N} / q^{0}$

Fig. 2. Chart and table of normalized pump discharge characteristic parameters $q_{N} / q^{0}$ 
На рис. 5 представлены графики переходных процессов при пуске и последующей вариации частоты вращения насоса для нефонтанирующей скважины, где выделены следующие временные моменты и состояния.

1. С пуском скважины из стационарного состояния останова при $t_{1}=20,005$ начинается заполнение НКТ жидкостью.

2. Момент полного заполнения НКТ и начала излива через устьевой штуцер в нефтесборник $t_{2}=20,042$. В данный момент имеет место скачкообразное снижение дебита в НКТ вследствие перепада давления на штуцере.

3. Понижение относительной частоты вращения вала насоса с 1 до 0,9 ед. в момент времени: $t_{3}=35$.
4. Восстановление частоты вращения вала насоса с 0,9 до 1 ед. в момент $t_{4}=36$.

5. Повышение относительной частоты вращения вала с 1 до 1,1 ед. при $t_{5}=37$.

6. В момент $t_{6}=37,72$ выход системы на условие «срыв подачи» по фактору избыточного содержания свободного газа у первой ступени насоса $\beta_{G N}>\beta_{G}^{L I M} \approx 0,25$.

Заметим, что все четыре временные шкалы графиков на рис. 4,5 имеют разные масштабы.

\section{Выводы}

1. Несмотря на упрощенный характер описания составных компонент системы, построенная модель (1)-(13) отражает целостную и логически связанную картину поведения скважины с

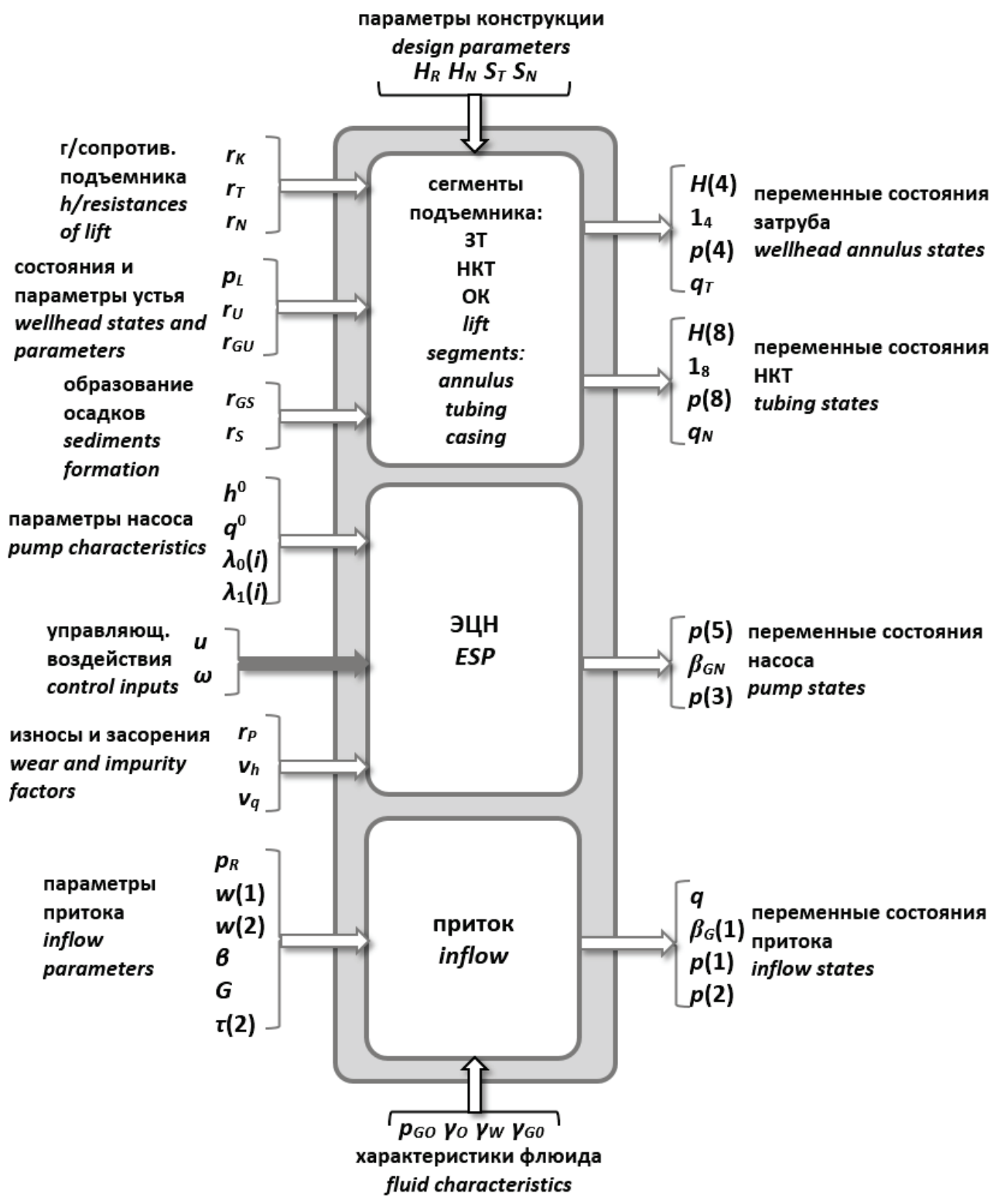

Рис. 3. Паралетрические настройки, возмущения и входо-выходные состояния модели

Fig. 3. Parametric settings, disturbances and input-output states of the model 


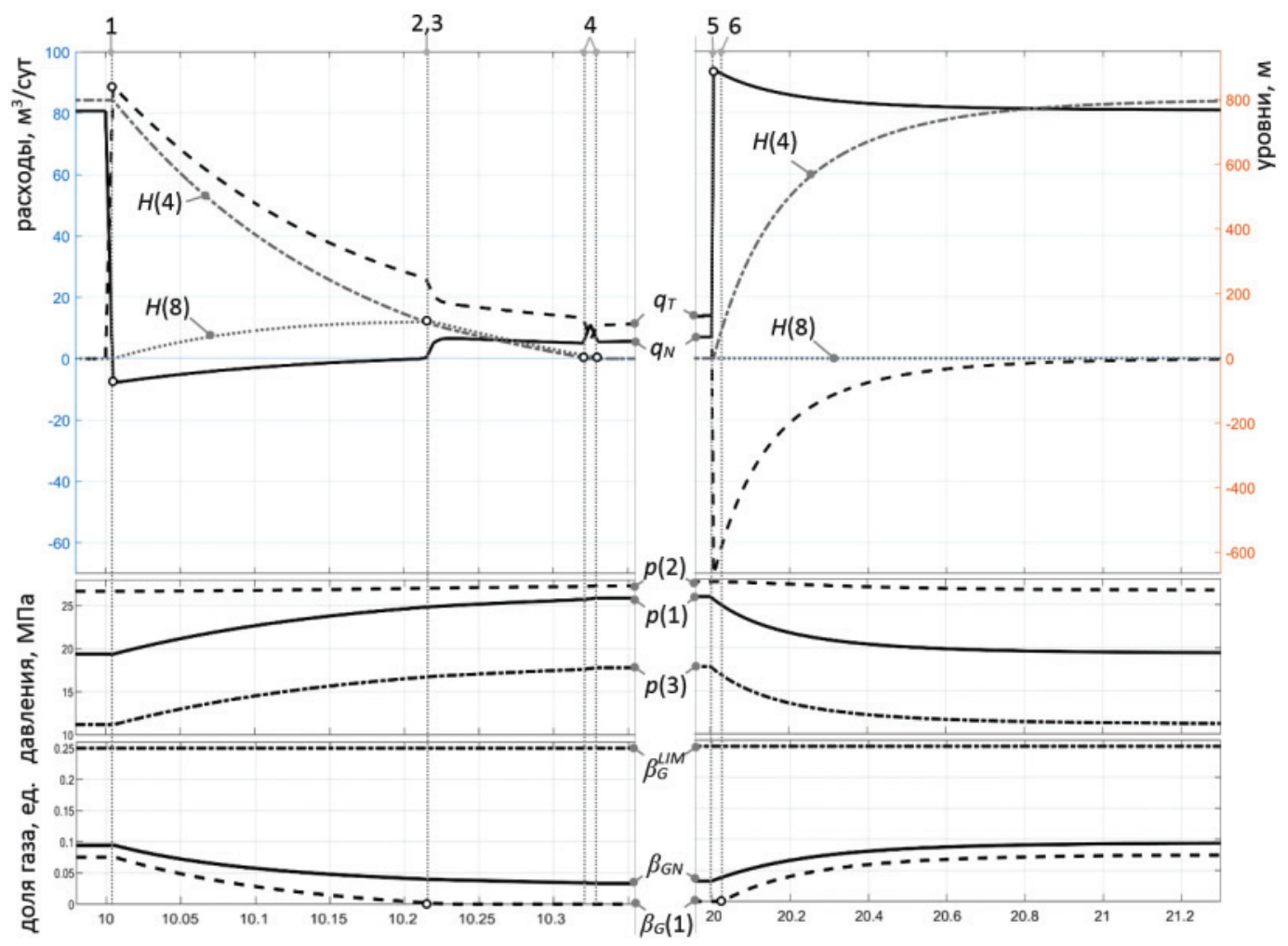

Рис. 4. Графики переходных процессов гидродинамических состояний в фонтанирующей скважине при пуске/останове

Fig. 4. Charts of hydrodynamic states transient processes in flowing well at start/stop

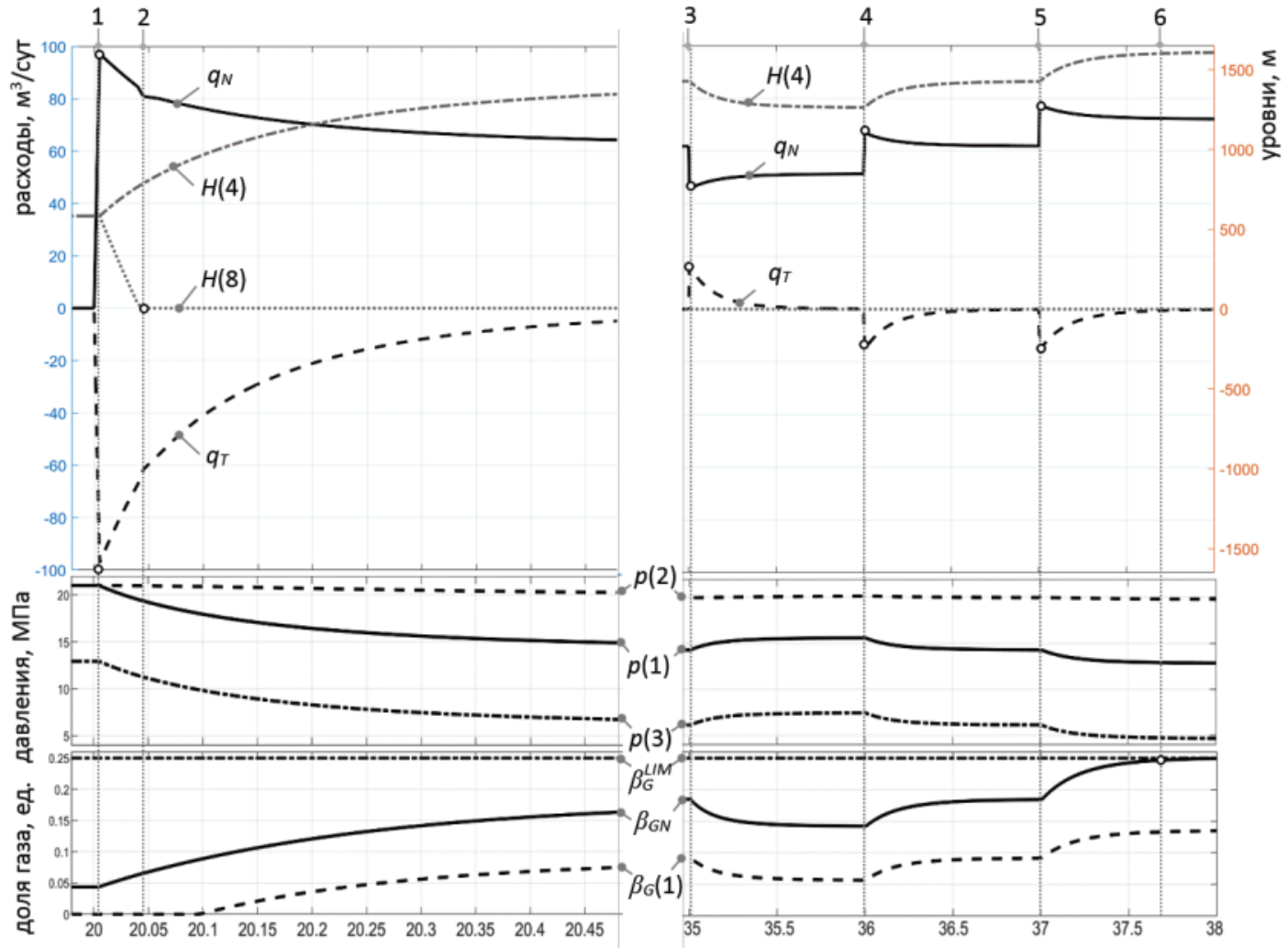

Pис. 5. Графики переходных процессов гидродинамических состояний в скважине без фонтана при смене частоты вращения насоса

Fig. 5. Charts of hydrodynamic states transient processes in a well without a fountain due to pump rotation frequency changes 
частотно-регулируемым ЭЦН в переходных и равновесных режимах эксплуатации.

2. Компонентная и факторная полнота математического описания гидродинамики системы является необходимым условием конструирования алгоритмов и регламентов параметрического оценивания $[15,16]$ по обновляемым данным промыслового контроля с последующим доопределением видов и уровней осложнений. Разработка схем устойчивого параметрического оценивания [20, 21] - одно из важных направлений развития рассмотренного решения.

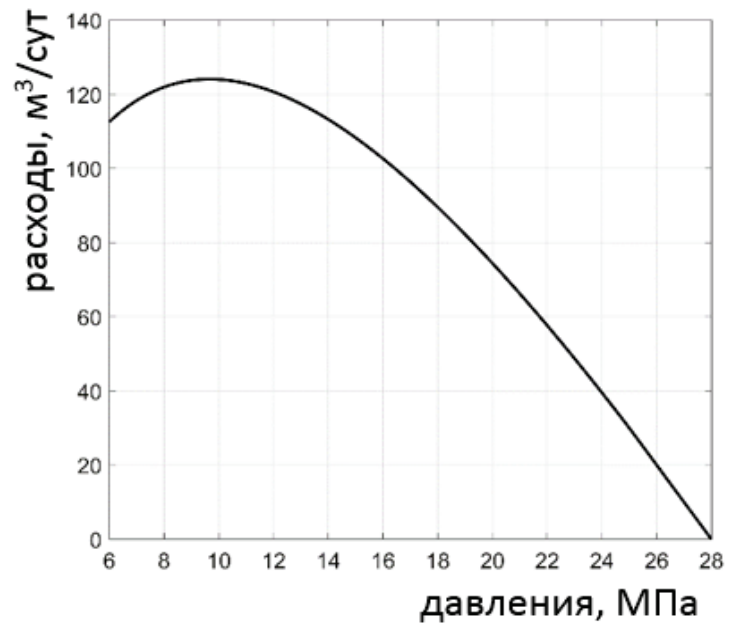

Рис. 6. Нелинейная кривая притока в условиях действия газового фактора на забое скважины

Fig. 6. Nonlinear inflow curve under the conditions of the gas factor at the well bore-hole

\section{СПИСОК ЛИТЕРАТУРЫ}

1. Haberer S., Krasikov A., Lavrinenko A. ESP monitoring and control system implementation in Western Siberia Brownfield // SPE Russian oil and gas exploration \& production technical conference and exhibition. - 2014. URL: https://doi.org/ 10.2118/171151-MS (дата обращения: 01.11.2018).

2. Автоматизация сбора и подготовки данных (в) TMС для проведения гидродинамических исследований скважин с использованием "виртуального расходомера» / А.А. Пашали, М.А. Александров, А.Г. Климентьев, И.Р. Ямалов, А.С. Топольников, А.В. Жонин, А.В. Колонских, В.Г. Михайлов // Нефтяное хозяйство. - 2016. - № 11. - С. 60-63.

3. Топольников А.С. Интеллектуальная обработка данных (В) TMC для интерпретации при проведении авто-ГДИС // Инженерная практика: Производственно-технический нефтегазовый журнал. - 2016. - Выпуск 10-11. URL: http://glavteh.ru/ авто-гдис-высокоточные-тмс / (дата обращения 11.07.2018).

4. Live well models a tool for production optimization / R.V. Rachapudi, B.Y. Haider, T. Al-Mutairi, K.W. Al Deyain, M. Al-Yahya, A. Shakeel, K.R. Qureshey, M. Harith // SPE Middle East intelligent energy conference and exhibition. - Manama, Bahrain, 28-30 October, 2013. URL: https://doi.org/10.2118/167469MS (дата обращения: 01.11.2018).

5. Real-time optimization approach for 15,000 ESP wells / S. Zdolnik, A. Pashali, D. Markelov, M. Volkov // Intelligent energy conference and exhibition. - 2008. URL: https://doi.org/ 10.2118/112238-MS (дата обращения: 01.11.2018).
3. Главная отличительная особенность предложенной модели от подобных решений [22-24] в явном учёте осложнений, вызванных действием газового фактора на забое скважины и ограничениями у приёма насоса. Нелинейная модель притока, в условиях падения забойного давления $p(1)$ ниже уровня насыщения $p_{G 0}$, определяет экстремальный вид функции расхода по жидкости (рис. 6), устанавливая тем самым физическое ограничение на максимально возможную производительность скважины.

4. Приведённые графики вычислительного анализа переходных режимов свидетельствуют о сложном характере движения жидкости в сегментах подъёмника, по сути скрытом до моментов начала излива на устье $t_{41}=10,324$ и $t_{41}=10,334$ - моменты начала изливов из 3Т и НКТ после выключения ЭЦН в фонтанирующей скважине (рис. 4) и $t_{2}=20,042$ - время заполнения НКТ после запуска ЭЦН в ранее остановленной нефонтанирующей скважине (рис. 5). Априорная оценка времени заполнения имеет важное значение для диагностики качества монтажа силовой установки и управления режимом освоения скважины после глушения.

5. Синхронный запуск и сопровождение вычислительного процесса по внешним контролируемым возмущениям в темпе с динамикой работы СC позволяет задействовать модель в режиме виртуального измерителя расширенного вектора состояния (рис. 3), некоторые компоненты которого: $q_{N}(t), q_{T}(t), \beta_{G N}(t), \beta_{G}(1, t)$, физически не удаётся контролировать в переходных режимах.

6. Сабиров А.А. Новые разработки в технике и технологии добычи нефти // Инженерная практика: Производственно-технический нефтегазовый журнал. - 2017. - Выпуск 01-02. URL: http://glavteh.ru/новые-разработки-в-технике-и-технолог/ (дата обращения 11.07.2018)

7. Extending ESP run life in gassy wells application / B. Kadio-Morokro, F. Curay, J. Fernandez, V. Salazar // SPE electric submersible pump symposium. - 2017. URL: https://doi.org/ 10.2118/185272-MS (дата обращения: 01.11.2018).

8. Honório W., Lemos D., De Lima R. ESP control and monitoring system of heavy oil Peregrino Field / SPE artificial lift conference. - 2015. URL: https://doi.org/10.2118/173929-MS (дата обращения: 01.11.2018).

9. PIPESIM. Steady-State Multiphase Flow Simulator. URL: http://www.software.slb.com/products/pipesim (дата обращения: 01.11.2018).

10. Petroleum Experts Integrated Production Modelling software. URL: http://www.petex.com (дата обращения: 01.11.2018).

11. Digital oilfield down under: implementation of an integrated production monitoring and management system for the Maari Field / A.J. Marron, M. Milner, A. 0'Hagan, S.S. Biniwale, R. Trivedi, T. Simpson, A.C. Tran // SPE/IATMI Asia pacific oil \& gas conference and exhibition. - 2015. URL: https://doi.org/ 10.2118/176485-MS (дата обращения: 01.11.2018).

12. Intelligent control system for gas-condensate field: a holistic automated smart workflow approach / V. Fomin, P. Kushmanov, P. Buryansh, M. Aksenov, N. Durygin, I. Solovyev, D. Govorkov, 
Y. Vedernikova, D. Iskakov // SPE Russian petroleum technology conference and exhibition. - 2016. URL: https://doi.org/ 10.2118/181986-MS (дата обращения: 01.11.2018).

13. Подземная гидромеханика / К.С. Басниев, Н.М. Дмитриев, Р.Д. Каневская, В.М. Максимов. - М.; Ижевск: Институт компьютерных исследований, 2005. - 496 с.

14. Подземная гидравлика / К.С. Басниев, А.М. Власов, И.Н. Кочина, В.М. Максимов. - М.: Недра, 1986. - 303 с.

15. Соловьев И.Г., Власов Д.А. Параметрическое оценивание модели гидродинамики плоскорадиального притока // Автоматизация, телемеханизация и связь в нефтяной промышленности. - 2012. - № 6. - С. 19-24.

16. Соловьев И.Г., Говорков Д.А. Идентификация гидродинамических параметров скважины оборудованной погружным насосом с наблюдателем процесса разгазирования нефти в подъемнике. Основы методики I // Автоматизация, телемеханизация и связь в нефтяной промышленности. - 2009. - № 3. C. $28-34$.

17. Соловьёв И.Г., Говорков Д.А., Ведерникова Ю.А. Режимы поведения скважин с ЭЦН при высоком газовом факторе // Автоматизация, телемеханизация и связь в нефтяной промышленности. - 2017. - № 6. - С. 28-34.

18. Метод снижения влияния свободного газа на эффективность работы скважинного насоса / К.Р. Уразаков, А.М. Азизов, И.А. Мухин, А.В. Щелоков // Нефтегазовое дело. - 2017. T.15. - № 2. - C. 58-62.

19. Хасанова Р.Д., Ивашов А.Ю., Вольцов А.А. Сепарационные установки для нефти с высоким газовым фактором // Инженер- ная практика: Производственно-технический нефтегазовый журнал. - 2016. - Вып. 12. URL: http://glavteh.ru/сепарационные-установки-нефть-газ / (дата обращения 11.07.2018).

20. Сергеев В.Л., Ву К.Д. Адаптивная интерпретация результатов гидродинамических исследований с учетом влияния ствола скважины // Известия Томского политехнического университета. Инжиниринг георесурсов. - 2016. - Т. 327. - № 9. - С. 70-77.

21. Боловин Е.В., Глазырин А.С. Метод идентификации параметров погружных асинхронных электродвигателей установок электроприводных центробежных насосов для добычи нефти // Известия Томского политехнического университета. Инжиниринг георесурсов. - 2017. - Т. 328. - № 1. - С. 123-131.

22. Соловьёв И.Г., Говорков Д.А. Задачи и инструменты анализа и управления режимами эксплуатации скважин // НТЖ Автоматизация, телемеханизация и связь в нефтяной промышленности. - 2013. - № 4. - С. 32-37.

23. Белашевский С.С., Говорков Д.А., Соловьёв И.Г. Регламент периодического обслуживания осложнённого фонда скважин // Автоматизация, телемеханизация и связь в нефтяной промышленности. - 2018. - № 1. - С. 21-26.

24. Solovyev I.G., Govorkov D.A. Mathematical model and computational analysis of hydrodynamics of the inflow to a horizontal segment-controlled well // Mathematical models and computer simulations - 2013. - V. 5. - № 1. - P. 1-6.

Поступила 07.11.2018 2.

\section{Информация об авторах}

Соловъев И.Г., кандидат технических наук, доцент, старший научный сотрудник Федерального исследовательского центра «Тюменский научный центр» СО РАН.

Говорков Д.А., кандидат технических наук, старший научный сотрудник Федерального исследовательского центра «Тюменский научный центр» СО РАН; доцент Тюменского индустриального университета.

Белашевский С.С., главный специалист 000 «Единые системы».

Ведерникова Ю.А., кандидат технических наук, доцент Тюменского индустриального университета. 
UDC 622.279:681.5

\title{
TRANSIENT PROCESSES MODEL OF A WELL WITH FREQUENCY-REGULATED ELECTRIC SUBMERSIBLE PUMP
}

Ilya G. Solovyev',

solovyev@ikz.ru

Denis A. Govorkov ${ }^{1,2}$,

dagovorkov@mail.ru

Sergey S. Belashevsky ${ }^{3}$,

s.belashevsky@gmail.com

\author{
Yulia A. Vedernikova ${ }^{2}$, \\ j_wedernik@mail.ru \\ 1 Tyumen Scientific Centre SB RAS, \\ 86, Malygin street, Tyumen, 625000, Russia. \\ 2 Tyumen Industrial University, \\ 38, Volodarsky street, Tyumen, 625000, Russia. \\ ${ }^{3}$ United system, \\ 4g, Kutateladze street, Novosibirsk, 630128, Russia.
}

The relevance of the research is related to the effectiveness of monitoring dynamic operating modes of wells equipped with a frequency-controlled electric submersible pump at development and computational implementation of well system models operating in real time. Complex functionality of the solution introduced in the article allows reproducing the systems real forms of behavior in transient and equilibrium operation modes under the impact of complicating factors, which is the basis of the correct parametric estimation using real-time control data of well operating states.

The main aim of the research is to construct a complex hydrodynamic model of the "reservoir-well-electric pump-wellhead» type, oriented to the tasks of complicated operating modes control of real-time systems.

objects: material balance, linear filtration, hydrostatics, linearized kinetics of friction losses, numerical modeling of differential equations.

Methods. The constructed and implemented hydrodynamic model of electric submersible pump-equipped well reproduces a holistic and logical «portrait» of actual behavior of the system in transient and equilibrium operation modes under the impact of complicating factors. The simplified nature of model components and connections descriptions allows the implementation and usage of functionally updated monitoring applications within the information resources of the enterprise.

Results. Complexity and factor wholeness of the model description are the basis for designing algorithms and regulations for parametric identification and subsequent recognition of the types and levels of impacting complications on system using actual operation data. The implementation of the computational process in sync with the dynamics of state and disturbances control data update make it possible to use the model as a virtual measure of the extended vector of well states in transient and steady-state operating modes.

\section{Key words:}

Inflow hydrodynamics, lift, electric centrifugal pump, model, hydrostatic head losses, productivity, complicating factors.

\section{REFERENCES}

1. Haberer S., Krasikov A., Lavrinenko A. ESP monitoring and control system implementation in Western Siberia Brownfield. SPE Russian oil and gas exploration \& production technical conference and exhibition, 2014. Available at: https://doi.org/10.2118/ 171151-MS (accessed 1 November 2018).

2. Pashali A.A., Aleksandrov M.A., Kliment'ev A.G., Yamalov I.R., Topolnikov A.S., Zhonin A.V., Kolonskikh A.V., Mikhaylov V.G. Automatization of collecting and preparation of telemetry data for well testing using «virtual flowmeter». Oil industry, 2016, no. 11 , pp. $60-63$. In Rus.

3. Topolnikov A.S. Intellektualnaya obrabotka dannykh (V) TMS dlya interpretatsii pri provedenii avto-GDIS [Intelligent processing of telemetry data for interpretation during automatic well tests]. Inzhenernaya praktika, 2016, no. 10-11. Available at: http://glavteh.ru/авто-гдис-высокоточные-тмс/ (accessed 11 July 2018).
4. Rachapudi R.V., Haider B.Y., Al-Mutairi T., Al Deyain K.W., Al-Yahya M., Shakeel A., Qureshey K.R., Harith M. Live well models a tool for production optimization. SPE Middle East intelligent energy conference and exhibition. Manama, Bahrain, 28-30 October, 2013. Availavle at: https://doi.org/ 10.2118/167469-MS (accessed 1 November 2018).

5. Zdolnik S., Pashali A., Markelov D., Volkov M. Real-time optimization approach for 15,000 ESP wells. Intelligent energy conference and exhibition. 2008. Availavle at: https://doi.org/ 10.2118/112238-MS (accessed 1 November 2018).

6. Sabirov A.A. Novye razrabotki v tekhnike i tekhnologii dobychi nefti [New developments in oil production technics and technology]. Inzhenernaya praktika, 2017, no. 01-02. Available at: http://glavteh.ru/новые-разработки-в-технике-и-технолог/ (accessed 11 July 2018).

7. Kadio-Morokro B., Curay F., Fernandez J., Salazar V. Extending ESP run life in gassy wells application. SPE electric submersible 
pump symposium. 2017. Available at: https://doi.org/ 10.2118/185272-MS (accessed 1 November 2018).

8. Honório W., Lemos D., de Lima R. ESP control and monitoring system of heavy oil Peregrino Field. SPE artificial lift conference. 2015. Available at: https://doi.org/10.2118/173929-MS (accessed 1 November 2018).

9. PIPESIM. Steady-State Multiphase Flow Simulator. Available at: http://www.software.slb.com/products/pipesim (accessed 1 November 2018).

10. Petroleum Experts Integrated Production Modelling software. Available at: http://www.petex.com (accessed 1 November 2018).

11. Marron A.J., Milner M., O’Hagan A., Biniwale S.S., Trivedi R., Simpson T., Tran A.C. Digital oilfield down under: implementation of an integrated production monitoring and management system for the Maari Field. SPE/IATMI Asia pacific oil \& gas conference and exhibition. 2015. Available at: https://doi.org/ 10.2118/176485-MS (accessed 1 November 2018).

12. Fomin V., Kushmanov P., Buryansh P., Aksenov M., Durygin N., Solovyev I., Govorkov D., Vedernikova Y., Iskakov D. Intelligent control system for gas-condensate field: a holistic automated smart workflow approach. SPE Russian petroleum technology conference and exhibition. 2016. Available at: https://doi.org/ 10.2118/181986-MS (accessed 1 November 2018).

13. Basniev K.S., Dmitriev N.M., Kanevskaya R.D., Maksimov V.M. Podzemnaya gidromekhanika [Underground hydromechanics]. Izhevsk, Institute of computer research, 2005. $496 \mathrm{p}$.

14. Basniev K.S., Vlasov A.M., Kochina I.N., Maksimov V.M. Podzemnaya gidraulika [Underground hydraulics]. Moscow, Nedra Publ., 1986. 303 p.

15. Solovyev I.G., Vlasov D.A. Parametric estimation of a hydrodynamic model of even-radial inflow. Automation, telemechanization and communication in oil industry, 2012, no. 6, pp. 19-24. In Rus.

16. Solovyev I.G., Govorkov D.A. Identification of hydrodynamic parameters of a well equipped with submersible pump using the observer of the oil degassing process in the lift. Basics of the methodology I. Automation, telemechanization and communication in oil industry, 2009, no. 3, pp. 28-34. In Rus.
17. Solovyev I.G., Govorkov D.A., Vedernikova Yu.A. Operating modes of ESP-equipped wells under high gas factor. Automation, telemechanization and communication in oil industry, 2017, no. 6, pp. 28-34. In Rus.

18. Urazakov K.R., Azizov A.M., Mukhin I.A., Shchelokov A.V. The method of reducing the influence of free gas on the performance of the downhole pump. Oil industry, 2017, vol. 15, no. 2, pp. 58-62. In Rus.

19. Khasanova R.D., Ivashov A.Yu., Voltsov A.A. Separatsionnye ustanovki dlya nefti s vysokim gazovym faktorom [Separating installations for oil with a high gas factor]. Inzhenernaya praktika, 2016, no. 12. Available at: http://glavteh.ru/сепарационныеустановки-нефть-газ/ (accessed 11 July 2018).

20. Sergeev V.L., Quang D.V. Adaptive interpretation of the results of hydrodynamic research considering a wellbore storage. Bulletin of the Tomsk Polytechnic University. Geo Assets Engineering, 2016, vol. 327, no. 9, pp. 70-77. In Rus.

21. Bolovin E.V., Glazyrin A.S. Method for identifying parameters of submersible induction motors of electrical submersible pump units for oil production. Bulletin of the Tomsk Polytechnic University. Geo Assets Engineering, 2017, vol. 328, no. 1, pp. 123-131. In Rus.

22. Solovyev I.G., Govorkov D.A. Tasks and tools of analysis and control of oil wells operational modes. Automation, telemechanization and communication in oil industry, 2013, no. 4, pp. 32-37. In Rus.

23. Belashevskiy S.S., Govorkov D.A., Solovev I.G. Regulation of complicated wells periodical service. Automation, telemechanization and communication in oil industry, 2018, no. 1, pp. 21-26. In Rus.

24. Solovyev I.G., Govorkov D.A. Mathematical model and computational analysis of hydrodynamics of the inflow to a horizontal segment-controlled well. Mathematical models and computer simulations, 2013, vol. 5, no. 1, pp. 1-6.

Received: 7 November 2018.

\section{Information about the authors}

Ilya G. Solovyev, senior researcher, Tyumen Scientific Centre SB RAS.

Denis A. Govorkov, senior researcher, Tyumen Scientific Centre SB RAS; associate professor, Tyumen Industrial University.

Sergey S. Belashevsky, main specialist, United system.

Yulia A. Vedernikova, associate professor, Tyumen Industrial University. 